2 Sparshatt A, Jones S, Taylor D. Quetiapine: dose-response relationship in schizophrenia. CNS Drugs 2008; 22: 49-68.

3 Arvanitis LA, Miller BG. Multiple fixed dose of 'Seroquel' (quetiapine) in patients with acute exacerbation of schizophrenia: a comparison with haloperidol and placebo. Biol Psychiatry 1997; 42: 233-46.

4 King DJ, Link CGG, Kowalcyk B. A comparison of bd and tid dose regimens of quetiapine (Seroquel) in the treatment of schizophrenia. Psychopharmacology 1998; 137: 139-46.

5 Barzega G, Bogetto F, Maina G, Ravizza L. Quetiapine in schizophrenic patients: a high- and low-dose double-blind comparison. Eur J Psychiatry 2000; 14: 221-32.

6 Emsley RA, Raniwalla J, Bailey PJ, Jones AM. A comparison of the effects of quetiapine ('Seroquel') and haloperidol in schizophrenic patients with a history of and a demonstrated, partial response to conventional antipsychotic treatment. Int Clin Psychopharmacol 2000; 15: $121-31$

7 Roy Chengappa KN, Parepally H, Brar JS, Mullen J, Shilling A, Goldstein JM. A random-assignment, double-blind, clinical trial of once- vs twicedaily administration of quetiapine fumarate in patients with schizophrenia or schizoaffective disorder: a pilot study. Can J Psychiatry 2003; 48: 187-94.

8 Conley RR, Kelly DL, Nelson MW, Richardson CM, Feldman S, Benham R, et al. Risperidone, quetiapine, and fluphenazine in the treatment of patients with therapy-refractory schizophrenia. Clin Neuropharmacol 2005; 28: 163-8.

9 Kahn RS, Schulz C, Palazov VD, Reyes EB, Brecher M, Svensson O, et al. Efficacy and tolerability of once-daily extended release quetiapine fumarate in acute schizophrenia: a randomized, double blind, placebocontrolled study. J Clin Psychiatry 2007; 68: 832-42.

10 Centre for Reviews and Dissemination. Undertaking Systematic Reviews of Research on Effectiveness: CRD's Guidance for Those Carrying Out or Commissioning Reviews. Centre for Reviews and Dissemination (CRD), Report 4 (2nd edn). CRD, 2001 (http://www.york.ac.uk/inst/crd/ report4.htm).

11 Buckley PF. Efficacy of quetiapine for the treatment of schizophrenia: a combined analysis of three placebo-controlled trials. Curr Med Res Opin 2004; 20: 1357-63.

\title{
Olive Tree community treatment centre for individuals with personality disorder: naturalistic service evaluation
}

\author{
Johannes L. Pretorius, ${ }^{1}$ Alberto Albeniz, ${ }^{2}$ Matthew Broome ${ }^{3}$
}

The Psychiatrist (2010), 34, 12-20, doi: 10.1192/pb.bp.108.023192

${ }^{1}$ Olive Tree Personality Disorders Unit, Coventry; ${ }^{2}$ Walsgrave Hospital,

Coventry; ${ }^{3}$ University of Warwick

Correspondence to

Johannes Pretorius

(wikus.pretorius@covwarkpt.nhs.uk)
Aims and method Community treatment for individuals with personality disorder is a fast developing field. We report here on the effectiveness of one such approach. We examine the referral pathway of all clients between January 2005 and April 2008, including the mean days spent in our unit, the days spent in a psychiatric hospital before and after admission to our unit, and the results of changes in the rating scales we routinely use.

Results Drop-out rates and the mean duration of therapy were acceptable. There has been a clear reduction of in-patient bed use and a small but significant improvement of most psychometric test results.

Clinical implications This study provides further evidence for the effectiveness of community treatment for individuals with personality disorder.

Declaration of interest None.
The Department of Health 2003 policy implementation guideline Personality Disorder: No Longer a Diagnosis of Exclusion ${ }^{1}$ set out to the UK's National Health Service (NHS) trusts the government's intentions for the delivery of personality disorder services within general mental health and forensic settings. In this document, the government built on standards four and five in the National Service Framework for Mental Health ${ }^{2}$ and set out specific guidance on the development of services for people with personality disorder. It made explicit that all trusts delivering general adult mental health services need to consider how to meet the needs of individuals with a personality disorder who experience significant distress or difficulty as a result of their disorder. Later in 2003, a further National Institute for Mental Health in England publication ${ }^{3}$ indicated that new funds would be made available to help stimulate the development of improved and new services to support users with personality disorders. The Olive Tree community treatment centre for individuals with personality disorder, created by the Coventry Primary Care Trust, became one of the pilots of this government initiative. ${ }^{4,5}$ At a time of change in the field of personality disorders, possible changes in classification and 
funding and the closing of a high-profile in-patient therapeutic community such as the Henderson, ${ }^{5}$ it may be of interest to readers to see how a community-based approach that would be easy to replicate in other venues has fared.

The Coventry Community Specialist Personality Disorder Service is a tertiary specialist team ${ }^{7}$ with the aim of using the structure of a clinical psychiatric service, designed around the assertive outreach recovery model, to deliver therapy in a variety of settings to individuals with a primary diagnosis of personality disorder. The service came into being towards the start of 2005, but owing to staff recruitment problems became fully operational only towards the end of the year. It provides a service to the local secondary mental health services: assessments, second opinions to psychiatrists, treatment of patients with a primary diagnosis of personality disorder, as well as training, supervision and mentoring of professionals involved in the care of such individuals. We also take an active role in the development of protocols and systems of communication about individuals with personality disorder, and training programmes on the diagnosis and treatment of such individuals.

There is mounting evidence that personality disorder can be successfully treated in the community with interventions such as therapeutic communities, ${ }^{8}$ partial hospitalisation ${ }^{9}$ and psychotherapy (metalisation-based therapy, ${ }^{10}$ dialectical behaviour therapy ${ }^{11}$ and cognitive analytic therapy ${ }^{12}$ ). The Coventry service takes the stance that the quality of the therapeutic alliance is one of the most important factors for successful outcome of psychotherapy ${ }^{13,14}$ and that a consistent, structured, focused, non-collusive approach based on the principles of attachment and the recovery model can be containing and therapeutic (see, for example, Bateman ${ }^{15}$ ).

Our service follows a team approach that has evolved with time ${ }^{16}$ and allows the provision of crisis support, mutual cover between therapists and good communication between different therapy modalities. The setting is a specialist unit situated away from the main psychiatric hospital in Coventry and therapists are expected to see their clients for individual work in the community, mostly at the user's general practice surgeries. Service users are expected to enter into both individual and group therapy, although individual therapy is offered to those who either do not need or cannot tolerate group therapy. We also offer a 'parallel service' of short-term group therapy without individual support or follow-up and with a much shorter period of assessment.

Group therapy ideally consists of long-term dynamic group therapy facilitated by two therapists. Therapy is structured around care plans that are frequently updated, meaningful and coherent to both client and therapists, and describe not only important, concrete goals and plans of action, including environmental manipulation, ${ }^{17}$ but also provide a reformulation of current psychotherapeutic core issues that take priority. This approach is based upon an integrationist approach as articulated by Albeniz \& Holmes. ${ }^{18}$ Core issues are identified early (some at assessment) in cooperation with the service user and addressed in therapy in a way designed as far as possible to suit the individual. The details of how these issues are addressed and how their evolution (and newly discovered issues) is further described are left to negotiation between the therapists and service user, provided the approach used is coherent to the therapists involved, the service user and supervisors. Therapy provision may also be adapted in terms of frequency, geographical location, style and content. Occasionally, elements of care such as crisis management or environmental manipulation may be handed over to other teams when it becomes apparent that these issues endanger the therapeutic alliance. Medication is prescribed by the doctors in the unit, or by the service user's current treating psychiatrist. ${ }^{19,20}$ Considerable time and effort is spent liaising with and influencing other parts of the mental health service involved in the care of our users.

Referrals (with a copy of patients' psychiatric files) are screened by a panel consisting of the potential assessors. The assessment process is led and supervised by the senior psychiatrists in the unit and is performed by the associate specialist (around $80 \%$ of all assessments) and senior therapists (predominantly one senior group therapist) who were trained after expressing an interest. The assessment consists of one to eight 1-hour long sessions, currently a mean of around $3 \mathrm{~h}$, guided by a semi-structured set of questionnaires, and results in a case summary that is prepared according to agreed guidelines. Admission into the unit depends on discussions between the assessors and the senior doctors, based upon the prepared case summaries and the questioning of the assessor, and decisions may occasionally be postponed pending further assessment sessions.

We aimed to create a flexible responsive service, thus:

1 The service would have a low drop-out rate and high retention rate; in those that did leave therapy, a mean of at least 6 months (150 days) of therapy would be achieved and $50 \%$ of discharged individuals would have completed therapy successfully.

2 Over the course of treatment there would be a reduction in wider mental health service use and an improvement in symptoms of personality disorder; over time after referral to the service, change would be shown in reduced hospital admissions and their duration, as well as an improvement in the psychometric measures we use in our unit.

\section{Method}

This paper presents the findings of a longitudinal cohort study as the result of a naturalistic service evaluation we completed after our unit has been operational for around 3 years. All referred clients between January 2005 and April 2008 were included in this study. All assessed clients were asked to sign a consent form to use their data for audit and research purposes, and so far all users who provided us with psychometric data have signed the consent forms.

\section{Data collection}

Inclusion criteria for the unit are:

- age between 18 and 65 years

- personality disorder as a primary diagnosis (meaning that although there might be evidence of other 
mental illness, personality disorder is seen as being the most important condition to address for the individual at the time of assessment; the presence of serious mental illness is therefore a relative rather than an absolute exclusion criterion).

Exclusion criteria are:

- being homeless

- significant forensic issues that cannot be safely contained in our unit

- very poor psychological mindedness

- insucient ego strength

- significant substance misuse that will disrupt therapy.

'Poor psychological mindedness' in our unit is located on the extreme end of the traditional psychological view of the concept, and is defined as an inability to accept and think about one's own role in the genesis and maintenance of problematic patterns of thinking, feeling and doing. The exclusion criterion of 'insufficient ego strength' is met when the post-assessment panel, after discussion with the assessor, judges the service user to be at risk of extreme impulsivity that would make it too dangerous to work with them, for example where hospitalisation is required, or judges them not to have the necessary inner resources to cope with even gentle explorative therapeutic approaches, therefore therapy would likely result in catastrophic decompensation. This criterion is very rarely used because assessment and treatment function as automatic screening processes, as individuals with very poor ego strength may find it difficult to apply themselves, although a patient, gentle, flexible approach may help to overcome hurdles in engagement.

\section{Study design}

All service users assessed for the full service are invited to complete a set of psychometric tests including the Personality Disorder Questionnaire version 4 (PDQ-4), ${ }^{21,22}$ Clinical Outcome Routine Evaluation (CORE), ${ }^{23}$ Social Anxiety and Distress Scale (SADS), ${ }^{24}$ Social Functioning Questionnaire $(\mathrm{SFQ})^{25}$ and a modified version of the Standardised Assessment of Personality - Abbreviated Scale (SAPAS). ${ }^{26}$ This last scale was originally designed to have eight items rated as 'yes' and 'no', but this was converted to a 10-point Likert scale to make it better suited to measure change. The psychometric tests reported here are routinely used to aid diagnosis and monitor progress, and the individual results are discussed with service users in each review clinic. These measures were also used by the Department of Health who monitored our performance as a pilot. Ethical committee approval was obtained and service users signed consent forms to use their anonymised data.

We planned to repeat the CORE six-monthly and the PDQ-4 yearly to improve the rate of completed scales but due to a low rate of repeating ratings we decided to repeat all the ratings yearly. All rating scales are self-report and were filled by service users on computer. A reduction of the score would show an improvement, except for the SFQ which should show an increase in the score. Additionally, our trust's information department provided us with 5 years' service use data on all users accepted into our unit at least a year ago, allowing us to compare mental health service use for at least 1 year after being accepted into our unit with data from before their admission.

\section{Data analysis}

Service use data from before and after admission were compared with the $\chi^{2}$-test. Analysis was done using SPSS for Windows, version 15

In the analyses of the psychometric test results, the Shapiro-Francia test and histograms were used to judge whether the scores for each subscale were normally distributed. When examining the difference in scores at assessment for those who responded and did not respond at follow-up, for those subscales that were normally distributed the two groups were examined using the unpaired $t$-test, and for those subscales that were not normally distributed the two groups were examined using the Mann-Whitney test.

In the set of analyses examining whether there was a change in scores over time for those users with scores on both occasions, for subscales where the change in scores was normally distributed the paired $t$-test was used to compare values between the two occasions, and for subscales where the change in scores was not normally distributed the Wilcoxon matched-pairs test was used to compare values between the two occasions. The significance of all results was judged on the $P$-value resulting from each test $(P<0.05$ is usually regarded as evidence of a statistically significant result). Only those psychometric tests were included in the analysis where the first measure was provided more than a year prior to April 2008.

\section{Results}

\section{Service user characteristics}

As can be seen from the referral pathway flowchart (Fig. 1), between January 2005 and April 2008 we had 501 referrals, 393 of which were for the full service and 108 for the 'parallel service'. There were 379 women referred (76\%), mean age 36 years (s.d. $=11.2$ ) and 122 men, average age 37 years (s.d. =10.8). Of the 337 individuals for whom we had ethnicity data, 327 (97\%) were White British, five Asian British, one Black British and four of mixed race. We accepted six (60\%) of those of Black and ethnic minority background into the service compared with 156 (48\%) of the White British individuals referred.

\section{Outcome of assessment}

At the time of analysis, 62 of all referred individuals were either still being assessed or have been invited for assessment appointments. We screened out 54 (11\%) of those referred for reasons such as the personality disorder not being the primary diagnosis, moving out of area, declining therapy, being referred to another service, or forensic issues. Of those individuals who did not complete the assessment procedure $(n=102,20 \%), 67$ never showed up after three appointment letters, 30 dropped out of assessment, and assessment was terminated early in cases where users moved out of the area or where it was 


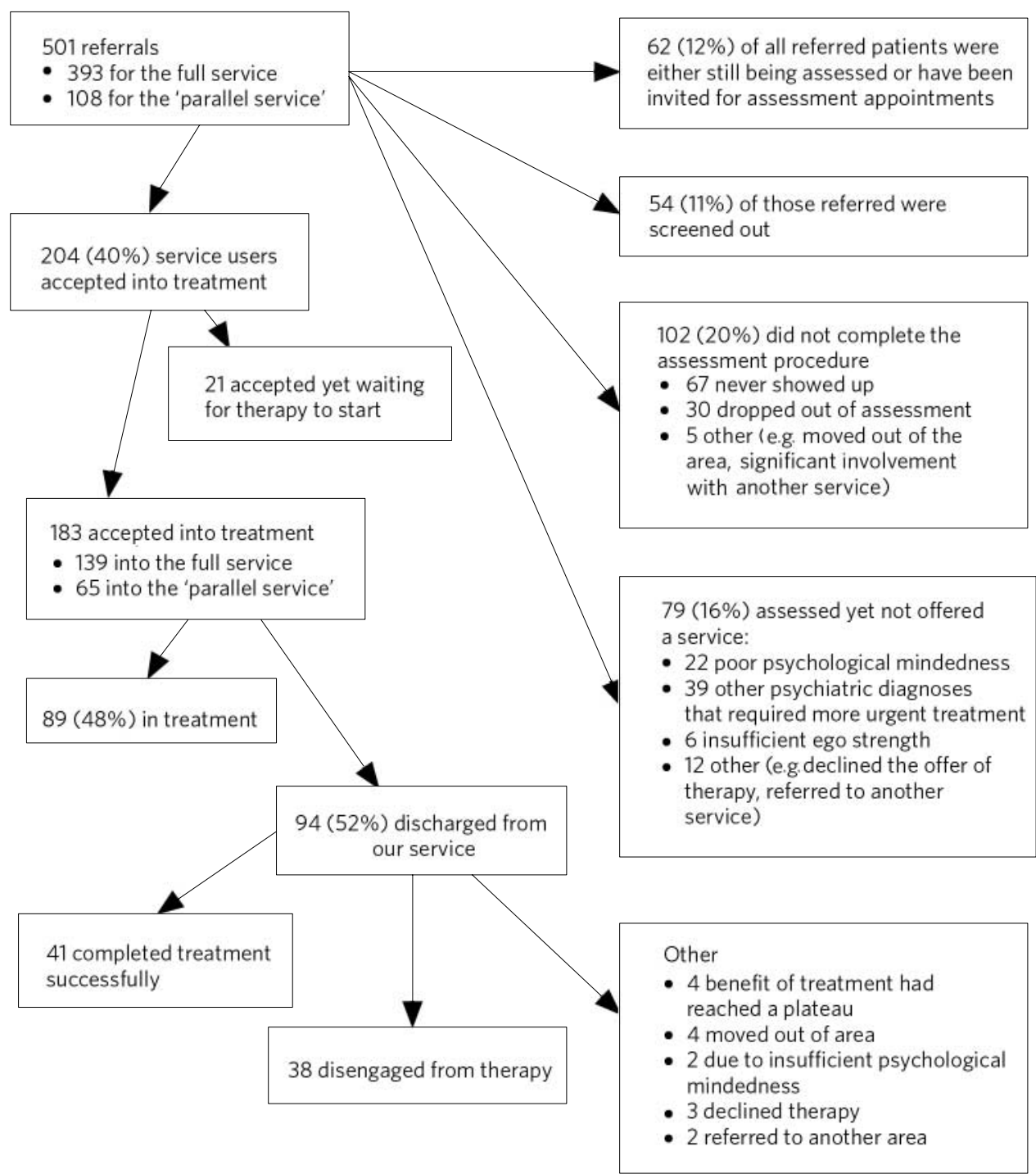

Fig 1 Referral pathway flowchart.

discovered they had significant involvement with another service. Some individuals $(n=79,16 \%)$ were assessed yet not offered a service, mainly for reasons of poor psychological mindedness $(n=22)$ and because there were other psychiatric diagnoses that required more urgent treatment $(n=39)$. Other reasons for not offering a service included the finding of insufficient ego strength in six individuals, and reasons such as declining the offer of therapy and being referred to another service.

Of 501 patients referred to our unit, $62(12 \%)$ are still in the assessment pathway, whereas 283 (64\% of the remainder) completed assessment. Of those assessed, 203 (72\%) have been accepted into our service and 94 have so far been discharged from therapy (41 classed as 'successful therapy' and 38 disengaged from therapy). The main reason for not accepting a referred client into our service seems to be that a high proportion (102 clients in total, or $23 \%$ of those who had an opportunity to undergo assessment) either never showed up or dropped out of the assessment process. There is no significant difference in age between those who dropped out and those who successfully completed therapy or those who are currently in therapy (all around 36 years old), but a significantly higher proportion of those who never showed up for the assessment were male (22 out of 67) compared with 18 out of 79 currently in therapy $\left(\chi^{2}=0.01\right)$ or 15 out of 79 screened out after assessment $\left(\chi^{2}=0.02\right)$. When comparing the gender mix and age of those screened out after having completed assessment $(n=79)$ with those accepted into the outreach service and for individual therapy $(n=79)$, no significant differences were found with the $\chi^{2}$-test ( 0.39 and 0.17 respectively), although the mean age of those screened out is slightly higher (40 v. 35.6 years).

A total of 204 service users were accepted into treatment (including 21 accepted yet waiting for therapy to start), 139 into the full service and 65 into the 'parallel service'. Of all those who were accepted and started treatment, $94(52 \%)$ users have so far been discharged from our service: 41 (43\% of all discharged) completed treatment successfully, $38 \quad(40 \%$ of all discharged $)$ 
disengaged from therapy, 4 discharged when we agreed (in supervision and the in the review clinic) that the benefit of treatment had reached a plateau, 4 moved out of area, 2 were found to have insufficient psychological mindedness, 3 declined therapy, and 2 were referred to another area.

The average number of treatment days in our unit (Table 1) for those who disengaged was 246 (s.d.=191); for successfully treated, 213 (s.d.=255); for those whose therapy plateaued, 485 (s.d. $=212$ ); those found to have insufficient psychological mindedness, 118 (s.d.=5); those who moved out of area, 432 (s.d. =93); and those referred to another service, 266 (s.d. = 170).

\section{In-patient days}

Table 2 gives the number of days spent as an in-patient on a psychiatric ward for 95 clients who were accepted into our service at least a year before the survey. The $\chi^{2}$-test of the average days spent in hospital for the 3 years before compared with 3 years after being accepted into our service is highly significant $(P=0.00)$. Comparing the year before being accepted into our service with the year following, there has been an $82 \%$ reduction of days spent in hospital and a $52 \%$ reduction in the number of admissions.

\section{Psychometric test results}

Initial rating scale results for service users assessed and accepted into the full service were compared for those who went on to provide a follow-up rating and those who did not (Table 3). An average of 86 users completed at least one scale before April 2007. Around $60 \%$ of those who completed the ratings on assessment went on to provide at least one follow-up rating. No significant differences on psychometric measures were found for most measures. Significant differences were found for the PDQ-4 subscales negativistic, histrionic and paranoid. A significant difference was also found for the SFQ. A high proportion (53\%) of ratings scored high on the PDQ-4 subscale of 'too good', indicating that the scores may underestimate the severity of pathology. In the PDQ-4, the lowest scores were found in the categories of histrionic (4\%), narcissistic (6\%) and antisocial traits (19\%), and the highest in avoidant (70\%), borderline $(61 \%)$, and impulsivity $(58 \%)$ subscales, whereas

\begin{tabular}{l} 
Table 1 Days spent in the Olive Tree centre (excluding 21 clients awaiting parallel therapy) \\
\cline { 3 - 6 } \\
\cline { 2 - 7 }
\end{tabular}

a. Service users in parallel therapy.

Table 2 Hospital admissions for patients accepted into the Olive Tree centre more than a year before the survey

\begin{tabular}{|c|c|c|c|c|c|c|c|c|}
\hline & \multirow{2}{*}{$\begin{array}{c}\text { Years } \\
\text { before } \\
\text { the survey }\end{array}$} & \multicolumn{2}{|c|}{ In-patient days, $n$} & \multirow{2}{*}{$\begin{array}{c}\text { Total } \\
\text { number of } \\
\text { admissions }\end{array}$} & \multirow{2}{*}{$\begin{array}{c}\text { Patients } \\
\text { in cohort } \\
n\end{array}$} & \multirow{2}{*}{$\begin{array}{l}\text { Total } \\
\text { number } \\
\text { of patients } \\
\text { applied to }\end{array}$} & \multirow{2}{*}{$\begin{array}{c}\text { Mean } \\
\text { days } \\
\text { per patient }\end{array}$} & \multirow{2}{*}{$\begin{array}{c}\text { Mean } \\
\text { admissions } \\
\text { per patient }\end{array}$} \\
\hline & & Total & s.d. & & & & & \\
\hline \multirow[t]{4}{*}{ Years before admission } & $3-4$ & 171 & 28.18 & 5 & & 95 & 1.80 & 0.05 \\
\hline & $2-3$ & 260 & 12.78 & 8 & & 95 & 2.74 & 0.08 \\
\hline & $1-2$ & 632 & 28.54 & 7 & & 95 & 6.65 & 0.07 \\
\hline & $0-1$ & 853 & 26.72 & 23 & & 95 & 8.98 & 0.24 \\
\hline \multirow{4}{*}{ Years since admission } & $0-1$ & 157 & 7.81 & 11 & 37 & 95 & 1.65 & 0.12 \\
\hline & $1-2$ & 140 & 14.02 & 4 & 44 & 58 & 2.41 & 0.07 \\
\hline & $2-3$ & 1 & & 0 & 14 & 14 & 0.07 & 0.00 \\
\hline & Total & 2214 & 22.59 & 58 & 95 & & 3.47 & 0.09 \\
\hline
\end{tabular}




\begin{tabular}{|c|c|c|c|c|c|}
\hline Scale & Subscale & $n$ & $\begin{array}{l}\text { Not responded at Time } \\
\text { 2, mean (s.d.) }\end{array}$ & $\begin{array}{c}\text { Responded at Time 2, mean } \\
\text { (s.d.) }\end{array}$ & $P$ \\
\hline \multirow[t]{17}{*}{ PDQ-4 } & Paranoid & 87 & $11.7(1.5)$ & $10.8(1.5)$ & $\star$ \\
\hline & Schizotypal & 86 & $13.8(2.2)$ & $13.4(2.5)$ & \\
\hline & Histrionic & 86 & $10.9(1.5)$ & $10.1(1.4)$ & * \\
\hline & Borderline & 87 & $13.0(2.0)$ & $12.8(1.6)$ & \\
\hline & Impulsivity & 88 & $8.7(1.6)$ & $9.1(1.6)$ & \\
\hline & Avoidant & 87 & $13(11,13.5)$ & $12(11,13)$ & \\
\hline & Antisocial & 87 & $9.0(1.6)$ & $8.8(1.6)$ & \\
\hline & Dependent & 87 & $11.9(2.1)$ & $11.3(2.0)$ & \\
\hline & Obsessive-compulsive & 88 & $12.3(1.6)$ & $11.8(1.7)$ & \\
\hline & Negativistic & 85 & $10.7(1.8)$ & $9.8(1.9)$ & * \\
\hline & Depressive & 86 & $12.2(1.5)$ & $12.6(1.3)$ & \\
\hline & Suspect & 88 & $2(2,2)$ & $2(2,2)$ & \\
\hline & Narcissistic & 88 & $11(11,13)$ & $10(10,12)$ & ** \\
\hline & Schizoid & 86 & $10(9,12)$ & $11(9.5,12)$ & \\
\hline & Conduct disorder & 87 & $17(15,19)$ & $16(15,18)$ & \\
\hline & Suspect & 88 & $2(2,2)$ & $2(2,2)$ & \\
\hline & Too good & 88 & $6.5(0.9)$ & $6.3(0.9)$ & \\
\hline \multirow[t]{5}{*}{ CORE } & Functions & 91 & $35.2(5.4)$ & $36.6(5.8)$ & \\
\hline & Well-being & 91 & $12(11,13)$ & $12(12,13)$ & \\
\hline & Problems & 90 & $51(43,54)$ & $47(40,54)$ & \\
\hline & Risk & 92 & $15(6,20)$ & $14(8,20)$ & \\
\hline & All items & 88 & $110(18)$ & $108(18)$ & \\
\hline SADS & & 87 & $193(55)$ & $199(48)$ & \\
\hline SFQ & & 89 & $16.1(5.1)$ & $18.0(4.5)$ & ** \\
\hline Modified SAPAS & & 95 & $52(46,62)$ & $54(46,60)$ & \\
\hline Satisfaction & & 41 & $73(63,77)$ & $73(66,77)$ & \\
\hline
\end{tabular}

${ }^{\star} P<0.05,{ }^{* \star} P<0.01$.

PDQ-4, Personality Disorder Questionnaire version 4; CORE, Clinical Outcome Routine Evaluation; SADS, Social Activities and Distress Scale; SFQ, Social Functioning Questionnaire; SAPAS, Standardised Assessment of Personality - Abbreviated Scale.

the rest ranged between 30 and $47 \%$. The fraction scoring within the clinical range of any scales on the CORE was 0.87 (s.d. =0.33) and 0.37 (s.d. $=0.48$ ) for the PDQ-4.

Psychometric ratings for those service users for whom we have at least one measure in addition to the rating done at assessment are presented in Table 4 . The mean number of days between measures is 300. Although all scales and subscales show an improvement in scores, changes at $5 \%$ significance are only for the CORE (all items, problems and risk subscales) and the PDQ-4 (borderline, depressive, schizoid, obsessive-compulsive subscales). Trait significance at $10 \%$ was observed in the SFQ, the functions subscale of the CORE and four subscales of the PDQ-4: paranoid, dependent, impulsivity and avoidant. Although there was an improvement in scores in the modified SAPAS, the SADS, the well-being subscale of the CORE (functions, well-being) and other subscales of the PDQ-4, this did not achieve statistical significance. There was little change in the PDQ-4 conduct disorder.

\section{Discussion}

We feel the evidence presented here suggests that our approach results in good engagement with an acceptable rate of discharge after successful therapy, even though we missed by $5 \%$ our target of $50 \%$ for discharge after successful therapy and mean duration of therapy in days markedly exceeded our target of 6 months. Bearing in mind the caveats described below, we feel that the evidence presented here suggests that our service had a broadly positive impact for our service users as shown in the outcomes from the psychometric tests used and has resulted in fewer days of hospital admission.

\section{Assessment non-attendance}

The relatively high number of referred clients who fail to attend for their first assessment appointment is an ongoing matter of concern and frustration, and perhaps more could be done to improve this. We continue to engage with referrers and we include information about our unit with all letters of invitation to the assessments. It is interesting that a disproportionate number of referred men never show up for their first appointment, but of those who do show up the drop-out rate appears to be low to normal. Although it can be speculated that the reason for this might be cultural and relates to stigma and male stereotyping, more needs to be understood about this finding.

\section{In-patient days}

The average duration of stay in our unit, even for those who eventually disengaged, is quite long in this difficult to engage population (246 days on average for those who dropped out of therapy). ${ }^{27}$ Furthermore, there has been a substantial reduction in the absolute and average number and duration of psychiatric hospital admissions. There is no control group against which to compare the size of this reduction and the trend seems to be an escalation of 


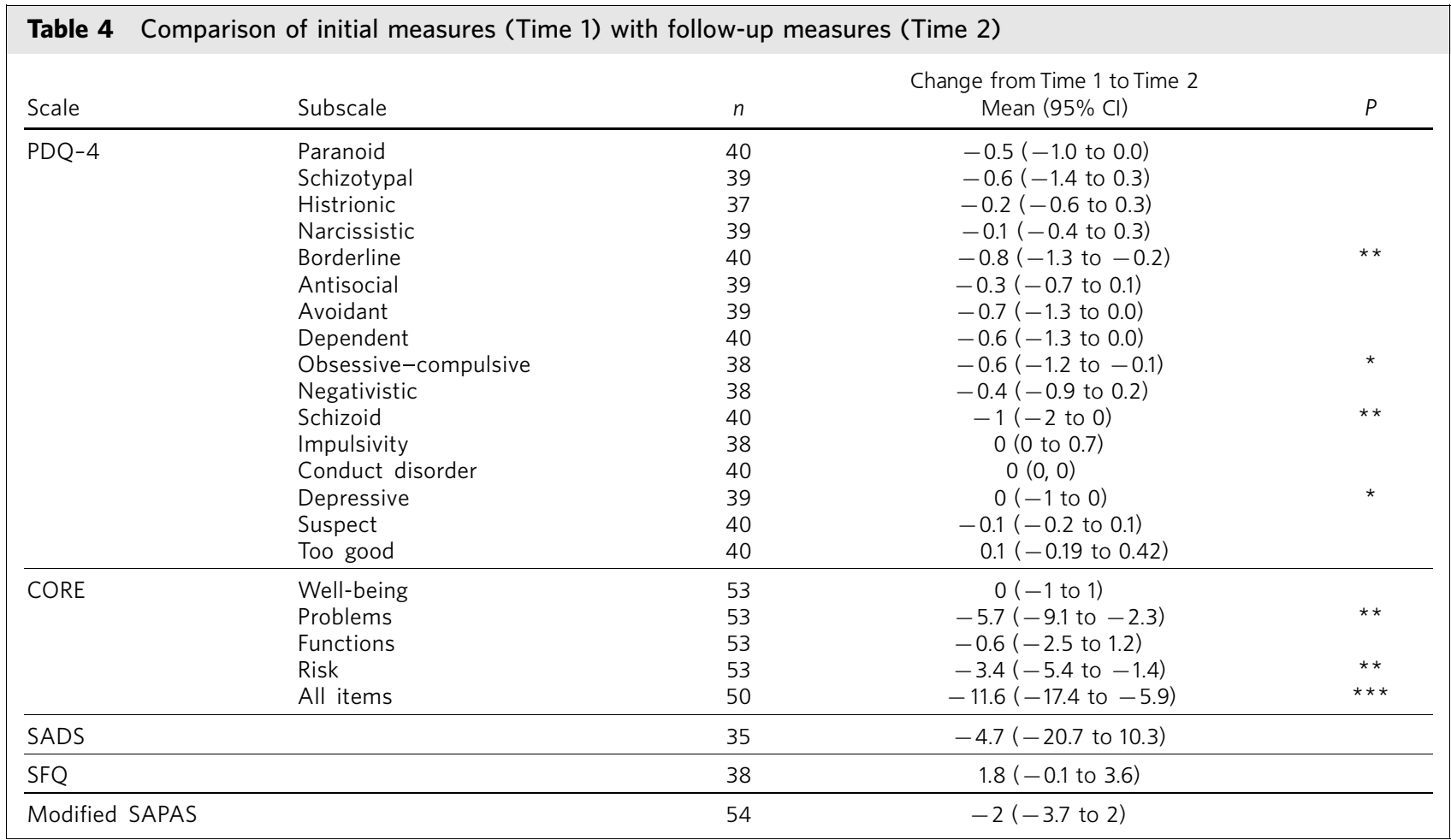

${ }^{*} P<0.05,{ }^{* \star} P<0.01,{ }^{* *} P<<0.001$.

PDQ-4, Personality Disorder Questionnaire version 4; CORE, Clinical Outcome Routine Evaluation; SADS, Social Activities and Distress Scale; SFQ, Social Functioning Questionnaire; SAPAS, Standardised Assessment of Personality - Abbreviated Scale.

hospital admissions leading up to patients being accepted into our unit, with a sudden reduction of hospital use. One possible explanation is that patients are referred to us in the midst or towards the end of an episode of serious mental illness (which is only a relative exclusion criterion in our unit), which starts to resolve immediately before or shortly after their acceptance into our unit. Another possibility, not mutually exclusive, is that users tend to be referred to us at a time that their support networks are in various stages of collapse, perhaps for a variety of reasons related to the consequences of their personality disorder and how this interacts with the particular circumstances of their lives, and stage of life. In the absence of a control group, our unit cannot take credit for the immediate reduction of hospital use, as this might be the consequence of work done shortly before the referral to our unit. We would propose that our direct involvement, both through our lengthy assessments before being accepted into our unit, as well as the containing, supportive and therapeutic effect we believe our involvement has, helps to hasten recovery from episodes of mental illness and facilitate communication and healthy therapeutic relationships between our users and other parts of the mental health service.

\section{Follow-up ratings}

We only have 33-59 follow-up ratings per user for around 100 users who are or have been in our unit for a year or more. This reduces the statistical power of the measurements to find a significant change, yet the vast majority of ratings showed an improvement, with statistically significant improvements in numerous measures. Perhaps paradoxically, those who completed a follow-up rating scored higher on the subscales paranoia, histrionic, negativism, narcissism and worse on the SFQ, implying that the sample who provided follow-up measures were slightly more disturbed. The personality traits that showed markedly below significant improvement were narcissistic and histrionic traits. Anecdotally, narcissistic traits do improve with time but require a longer period of attachment, so change may only manifest with longer follow-up. The approach to narcissism in our unit has changed over time and it will be of interest to see how this measure changes in the future. It is interesting that although impulsive traits did not show a significant improvement on the PDQ-4, this seems at odds with an improvement of the CORE subscales of problems and risk, and the SFQ, all of which would seem to require improved impulsivity. Although this finding is not understood, one possibility is that the subjective view of impulsivity in general terms, as measured by the PDQ-4, lags behind reality, which may be better captured by the CORE and SFQ. Also, our unit does not formally provide teaching or training in emotion regulation, leaving this aspect to the individual therapist to attend to when appropriate and potential improvement in this area may be sacrificed for work in other areas.

Of interest are the clearly positive changes seen in 'difficult to treat' traits such as schizoid or anankastic traits. 
It is also interesting that the CORE well-being subscale, as well as the SADS, do not show a significant change, in contrast with the SFQ and the aggregate CORE, both of which show positive change. It appears from this that although improvement occurs in most measured areas, the subjective level of stress remains more or less constant. One interpretation of this may be that users are able to 'push' themselves in therapy, building new skills and experiences in preference to distress reduction. It should also be borne in mind that the psychometric tools we use can only be imperfect proxy measures of hypothesised underlying pathology and the dramatic change in the schizoid subscale, for example, may be measuring something other than true schizoid personality disorder pathology.

The modified SAPAS shows only a trend towards significance. This short rating scale may not be as sensitive as the more detailed rating scales for measuring actual or subjective change in personality pathology, and changes in this scale, as with compulsivity on the PDQ-4, may lag behind more sensitive measures.

\section{Future directions}

It is humbling to realise that the mean reduction in ratings of subscales was 5\% both on the PDQ-4 (from 34 to 29\%) and the CORE (from 98 to 93\%). The wide standard deviation of scores on these measures indicate that there may only be a small proportion of service users who are sufficiently close to the cut-off mark on these tests to cross the boundary between being in the normal and clinical range, even with moderate improvement. There are service users who did substantially better than others and some individuals need longer to change their lives than others, therefore a future description of these subgroups might be of interest. Ideally, other indicators of successful therapy such as rates of self-harm, employment status, average annual income and other measures would be included in this paper. We have direct feedback from service users, provided individually and in forums, but this is not included in this article.

It may be important to compare our approach with other treatment models. We are also continuing to monitor progress with all the measures we presented in this paper in order to evaluate longer-term outcomes, including a more accurate idea of change and correlations between variables as hypothesised. The numbers of referred clients are also not an accurate reflection of our workload, as we only started to accept routine referrals from the end of 2005 onwards, and we have had an increased rate of referrals and completed assessments year on year so far.

\section{Acknowledgement}

We thank Professor Scott Weich for his invaluable input and support.

\section{About the authors}

Johannes L. Pretorius is Associate Specialist in Psychotherapy, Olive Tree Personality Disorders Unit, Swanswell point-Community Mental Health Services, Coventry. Alberto Albeniz is Consultant Psychiatrist in Psychotherapy, Lead Clinician for Coventry Personality Disorder Services,
Caludon Centre, Walsgrave Hospital, Coventry. Matthew Broome is Associate Clinical Professor of Psychiatry, Honorary Consultant Psychiatrist in Early Intervention, Health Sciences Research Institute, University of Warwick.

\section{References}

1 National Institute for Mental Health in England. Personality Disorder: No Longer a Diagnosis of Exclusion - Policy Implementation Guidance for the Development of Services for People with Personality Disorder. Department of Health, 2003.

2 Department of Health. National Service Framework for Mental Health: Modern Standards and Service Models. Department of Health, 1999.

3 National Institute for Mental Health in England. Breaking the Cycle of Rejection: The Personality Capabilities Framework. NIMHE, 2003.

4 Crawford MJ, Price K, Rutter D, Moran P, Tyrer P, Bateman A, et al. Dedicated community-based services for adults with personality disorder: Delphi study. Br J Psychiatry 2008; 193: 342-3.

5 Crawford $M$, Rutter $D$, Price $K$, Weaver $T$, Josson $N$, Tyrer $P$, et al. Learning the Lessons: A Multi-Method Evaluation of Dedicated CommunityBased Services for People with Personality Disorder. National Coordinating Centre for NHS Service Delivery and Organisation, 2007.

6 Mayor S. MPs call on health minister to halt closure of hospital for personality disorders. BMJ 2008; 336: 181.

7 Bateman AW, Tyrer P. Services for personality disorder: organisation for inclusion. Adv Psychiatr Treat 2004; 10: 425-33.

8 Chiesa M, Fonagy $P$, Holmes J. Six-year follow-up of three treatment programs to personality disorder. J Pers Disord 2006; 20: 493-509.

9 Bateman A, Fonagy P. Health service utilization costs for borderline personality disorder patients treated with psychoanalytically oriented partial hospitalization versus general psychiatric care. Am J Psychiatry 2003; 160: 169-71.

10 Bateman A, Fonagy P. Mentalization based treatment of borderline personality disorder. J Pers Disord 2004; 18: 36-51.

11 Linehan MM, Comtois KA, Murray AM, Brown MZ, Gallop RJ, Heard $\mathrm{HL}$, et al. Two-year randomized controlled trial and follow-up of dialectical behavior therapy vs therapy by experts for suicidal behaviors and borderline personality disorder. Arch Gen Psychiatry 2006; 63: 757-66.

12 Ryle A, Golynkina K. Effectiveness of time-limited cognitive analytic therapy of borderline personality disorder: factors associated with outcome. Br J Med Psychol 2000; 73: 197-210.

13 Krupnick JL, Sotsky SM, Simmens S, Moyer J, Elkin J, Watkins J, et al. The role of the therapeutic alliance in psychotherapy and pharmacotherapy outcome: findings in the National Institute of Mental Health Treatment of Depression Collaborative Research Program. J Consult Clin Psychol 1996; 64: 532-9.

14 Stiles WB, Agnew-Davies R, Hardy GE, Barkham M, Shapiro DA Relations of the alliance with psychotherapy outcome: findings in the second Sheffield Psychotherapy Project. J Consult Clin Psychol 1998; 66: 791-802.

15 Bateman, AW. Integration in psychotherapy: an evolving reality in personality disorder. Br J Psychother 2000; 17: 147-56.

16 Pretorius JL, Albeniz A. Learning experience from the Coventry Community Personality Disorder Service. Br J Forensic Pract 2006; 8: 34-40.

17 Tyrer P, Bajaj P. Nidotherapy: making the environment do the therapeutic work. Adv Psychiatr Treat 2005; 11: 232-8.

18 Albeniz A, Holmes J. Psychotherapy integration: its implications for psychiatry. Br J Psychiatry 1996; 169: 563-70.

19 Solof P. Algorithms for pharmacological treatment of personality dimensions. Symptom specific treatments for cognitive-perceptual, affective, and impulse-behavioural dysregulation. Bull Meninger Clin 1998; 62: 195-214.

20 Tyrer P, Bateman AW. Drug treatment for personality disorders. Adv Psychiatr Treat 2004; 10: 389-98. 
21 Hyler SE, Rieder RO, Williams JBW, Spitzer RL, Hendler J, Lyons M. The Personality Diagnostic Questionnaire: development and preliminary results. J Pers Disord 1988; 2: 229-37.

22 Hyler SE, Skodol AE, Kellman HD, Oldham J, Rosnick L. The validity of the Personality Diagnostic Questionnaire: a comparison with two structured interviews. Am J Psychiatry 1990; 147: 1043-8.

23 Barkham M, Evans C, Margison F, Mcgrath G, Mellor-Clark J, Milne, et al. The rationale for developing and implementing core outcome batteries for routine use in service settings and psychotherapy outcome research. J Ment Health 1998; 7: 35-47.

24 Watson D, Friend R. Measurement of social-evaluative anxiety. J Consult Clin Psychol 1969; 33: 448-57.
25 Tyrer P, Nur U, Crawford M, Karlsen S, MacLean C, Rao B, et al. The Social Functioning Questionnaire: a rapid and robust measure of perceived functioning. Intern J Social Psychiatr 2005; 51: 265-75.

26 Moran $P$ Leese $M$, Lee $T$, Walters $P$, Thornicroft $G$ Mann $A$. Standardised Assessment of Personality - Abbreviated Scale (SAPAS): preliminary validation of a brief screen for personality disorder. Br J Psychiatry 2003; 183: 228-32.

27 Gunderson JG, Prank AF, Ronningstam EF, Watcher S, Lynch V, Wolf PJ. Early discontinuance of borderline patients from psychotherapy. J Nerv Ment Dis 1989; 177: 38-42.

\title{
Service user, patient, client, user or survivor: describing recipients of mental health services
}

\author{
Peter Simmons, ${ }^{1}$ Chris J. Hawley, ${ }^{1,2}$ Tim M. Gale, ${ }^{1}$ Thanusha Sivakumaran $^{1}$
}

The Psychiatrist (2010), 34, 20-23, doi: 10.1192/pb.bp.109.025247

${ }^{1}$ Hertfordshire Partnership NHS Foundation Trust, Queen Elizabeth II Hospital, Welwyn Garden City, Hertfordshire; ${ }^{2}$ Division of Psychology, University of Hertfordshire

Correspondence to Peter Simmons (peter.simmons@hertspartsft.nhs.uk)

\begin{abstract}
Aims and method To determine which terms receivers of mental health services wish to be known by (service user, patient, client, user, survivor) according to the professional consulted (psychiatrist, nurse, psychologist, social worker, occupational therapist). We conducted a questionnaire study to assess terms by like or dislike and by rank order. There were 336 participants from local catchment area secondary care community and in-patient settings in east Hertfordshire.
\end{abstract}

Results Patient is the preferred term when consulted by psychiatrists and nurses, but it is equally preferable to client for social workers and occupational therapists. Service user is disliked more than liked overall, particularly by those who consulted a health professional, but not by those who consulted a social worker. A significant minority wish to be regarded as a survivor or user.

Clinical implications National and local mental health services should adopt evidence-based terminology in referring to 'patient' or, in some groups, 'patient or client' in preference to 'service user'.

Declaration of interest None.
Various terms are used to describe receivers of health services. The General Medical Council refers to people treated by a doctor as patients; ${ }^{1}$ the Nursing and Midwifery Council refers to people, patients/clients and clients/ patients; ${ }^{2}$ the British Association of Social Workers refers to service users; ${ }^{3}$ the British Psychological Society (in Code of Ethics and Conduct) and the College of Occupational Therapists both refer to clients. ${ }^{4,5}$ A former chairman of the Patients' Association suggested alternatives to patient, considering the terms client, consumer and user, with an overall preference for the term user. ${ }^{6}$

The National Service Framework for Mental Health refers to service user 48 times, patient 12 times and client twice. ${ }^{7}$ The Royal College of Psychiatrists, in its report Mental Illness: Stigmatisation and Discrimination within the Medical Profession, refers to patient 77 times, service user 7 times and user (independently of service user) twice. ${ }^{8}$ Of national mental health charities, SANE refers to 'people affected by mental illness' (www.sane.org.uk/ AboutSANE), whereas MIND variously refers to user, service user or user/survivor. ${ }^{9}$

In a study of adult psychiatric out-patient clinic attendees in London, Ritchie et al reported that of those expressing a preference for patient or client (96\%) rather than 'other', $77 \%$ favoured patient and $23 \%$ client, whereas $47 \%$ disliked client and $14 \%$ disliked patient. ${ }^{10}$

In another London study, 133 people who received care from care coordinators from community mental health centres were asked about their preference of the terms patient, client or service user when being addressed by different mental health professionals. ${ }^{11}$ The survey indicated that between 7 and $10 \%$ wished to be addressed as a service user, 41 to $75 \%$ as a patient, and 17 to $47 \%$ as a client, the percentage varying according to the different professional 